Acta Crystallographica Section E

Structure Reports

Online

ISSN 1600-5368

\section{Chlorido $\left\{N^{2}, N^{6}\right.$-dibenzyl- $N^{2}, N^{6}$-bis- [(diphenylphosphanyl)methyl]pyridine- 2,6-diamine]methylplatinum(II)}

\section{Zi-Jia Wang, Xiao-Xi Wang and Chong-Qing Wan*}

Department of Chemistry, Capital Normal University, Beijing 100048, People's Republic of China

Correspondence e-mail: wanchqing@yahoo.com.cn

Received 17 September 2010; accepted 24 September 2010

Key indicators: single-crystal X-ray study; $T=293 \mathrm{~K}$; mean $\sigma(\mathrm{C}-\mathrm{C})=0.008 \AA$; $R$ factor $=0.024 ; w R$ factor $=0.050 ;$ data-to-parameter ratio $=14.8$.

In the title mononuclear complex, $\left[\mathrm{Pt}\left(\mathrm{CH}_{3}\right) \mathrm{Cl}\left(\mathrm{C}_{45} \mathrm{H}_{41} \mathrm{~N}_{3} \mathrm{P}_{2}\right)\right]$, the pyridine-2,6-diamine ligand can be viewed as a centrosymmetric motif having two pendant $N$-benzyl- $N$-[(diphenylphosphanyl)methyl] arms, the two $\mathrm{P}$ atoms of which chelate to the $\mathrm{Pt}^{\mathrm{II}}$ ion, forming a ten-membered metallocycle. A distorted square-planar coordination geometry around the $\mathrm{Pt}^{\mathrm{II}}$ atom is completed by a methyl ligand and a chloride ion. The packing between the mononuclear units is achieved through $\mathrm{C}-\mathrm{H} \cdots \pi$ interactions, which link the molecules into chains along the $c$ axis.

\section{Related literature}

For coordination complexes with hemilabile tridentate ligands with $\mathrm{P} X \mathrm{P}(X=\mathrm{C}, \mathrm{N}, \mathrm{O}, \mathrm{S}$, and As) donor sets, see: Ainscough et al. (2004); Song et al. (2002); Kunz et al. (2010); Wang et al. (2010); Zhang \& Cheng (1996). For a coordination complex of the 2,6-bis $(N$-benzyl- $N$-diphenylphosphinomethylamino)pyridine ligand, see: Li et al. (2005). For $\mathrm{C}-\mathrm{H}$ (benzene) $\cdots \pi$ interactions, see: Umezawa et al. (1998).

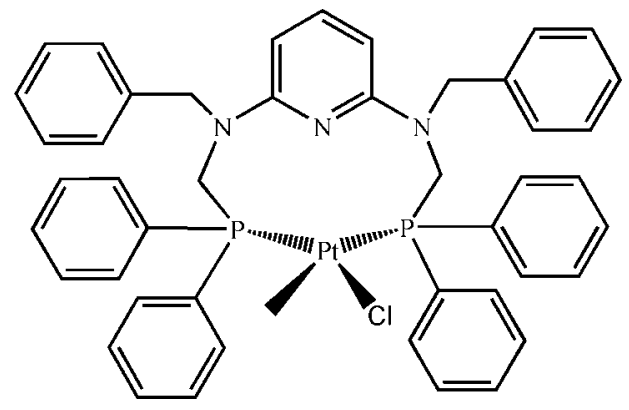

\section{Experimental}

Crystal data

$\left[\mathrm{Pt}\left(\mathrm{CH}_{3}\right) \mathrm{Cl}\left(\mathrm{C}_{45} \mathrm{H}_{41} \mathrm{~N}_{3} \mathrm{P}_{2}\right)\right]$

$M_{r}=931.32$

Orthorhombic, $P 2_{1} 2_{1} 2_{1}$

$a=15.3515(9) \AA$

$b=15.9294(10) \AA$

$c=16.5197(10) \AA$

$V=4039.7(4) \AA^{3}$

$Z=4$

Mo $K \alpha$ radiation

$\mu=3.66 \mathrm{~mm}^{-1}$

$T=293 \mathrm{~K}$

$0.32 \times 0.24 \times 0.16 \mathrm{~mm}$

\section{Data collection}

Bruker APEXII CCD area-detector diffractometer

Absorption correction: multi-scan

(SADABS; Bruker, 2007)

$T_{\min }=0.640, T_{\max }=1.000$

Refinement

$R\left[F^{2}>2 \sigma\left(F^{2}\right)\right]=0.024$

$w R\left(F^{2}\right)=0.050$

$S=0.97$

7108 reflections

479 parameters

$\mathrm{H}$-atom parameters constrained

21886 measured reflections 7108 independent reflections 6498 reflections with $I>2 \sigma(I)$ $R_{\text {int }}=0.029$

Table 1

Selected bond lengths $(\AA)$.

\begin{tabular}{llll}
\hline $\mathrm{Pt} 1-\mathrm{C} 46$ & $2.172(3)$ & $\mathrm{Pt} 1-\mathrm{P} 2$ & $2.3153(10)$ \\
$\mathrm{Pt} 1-\mathrm{P} 1$ & $2.2105(11)$ & $\mathrm{Pt} 1-\mathrm{Cl} 1$ & $2.3663(12)$ \\
\hline
\end{tabular}

Table 2

Hydrogen-bond geometry $\left(\AA,^{\circ}\right)$.

$C g 1$ is the centroid of the $\mathrm{C} 15-\mathrm{C} 20$ benzene ring.

\begin{tabular}{lllll}
\hline$D-\mathrm{H} \cdots A$ & $D-\mathrm{H}$ & $\mathrm{H} \cdots A$ & $D \cdots A$ & $D-\mathrm{H} \cdots A$ \\
\hline $\mathrm{C} 5-\mathrm{H} 5 \cdots C g 1^{\mathrm{i}}$ & 0.93 & 3.00 & $3.812(2)$ & 146 \\
$\mathrm{C} 26-\mathrm{H} 26 B \cdots C g 1^{\mathrm{ii}}$ & 0.97 & 2.96 & $3.807(2)$ & 147 \\
\hline
\end{tabular}

Symmetry codes: (i) $-x+\frac{3}{2},-y+1, z+\frac{1}{2}$; (ii) $-x+\frac{3}{2},-y+1, z-\frac{1}{2}$.

Data collection: APEX2 (Bruker, 2007); cell refinement: APEX2 and $S A I N T$ (Bruker, 2007); data reduction: $S A I N T$; program(s) used to solve structure: SHELXS97 (Sheldrick, 2008); program(s) used to refine structure: SHELXL97 (Sheldrick, 2008); molecular graphics: SHELXTL (Sheldrick, 2008); software used to prepare material for publication: SHELXTL and PLATON (Spek, 2009).

The authors are grateful for financial support from the Science and Technology program, Beijing Municipal Education Commission.

Supplementary data and figures for this paper are available from the IUCr electronic archives (Reference: BQ2234).

\section{References}

Ainscough, E. W., Brodie, A. M., Burrell, A. K., Derwahl, A., Jameson, G. B. \& Taylor, S. K. (2004). Polyhedron, 23, 1159-1168

Bruker (2007). APEX2, SAINT and $S A D A B S$. Bruker AXS Inc., Madison, Wisconsin, USA.

Flack, H. D. (1983). Acta Cryst. A39, 876-881. 


\section{metal-organic compounds}

Kunz, P. C., Wetzel, C., Bongartz, M., Noffke, A. L. \& Spingler, B. (2010). J. Organomet. Chem. 695, 15-16.

Li, Q. S., Wan, C. Q., Xu, F. B., Song, H. B. \& Zhang, Z. Z. (2005). Inorg. Chim. Acta, 358, 2283-2291.

Sheldrick, G. M. (2008). Acta Cryst. A64, 112-122.

Song, H. B., Zhang, Z. Z. \& Mak, T. C. W. (2002). Inorg. Chem. Commun. 5, $442-445$
Spek, A. L. (2009). Acta Cryst. D65, 148-155.

Umezawa, Y., Tsuboyama, S., Honda, K., Uzawa, J. \& Nishio, M. (1998). Bull. Chem. Soc. Jpn, 71,1202-1213.

Wang, X. B., Feng, J. Z., Huang, J., Zhang, J. Y., Pan, M. \& Su, C. Y. (2010). CrystEngComm, 12, 725-729.

Zhang, Z. Z. \& Cheng, H. (1996). Coord. Chem. Rev. 147, 1-39. 


\section{supporting information}

Acta Cryst. (2010). E66, m1341-m1342 [doi:10.1107/S1600536810038134]

\section{Chlorido $\left\{N^{2}, N^{6}\right.$-dibenzyl- $N^{2}, N^{6}$-bis [(diphenylphosphanyl)methyl]pyridine-2,6- diamine\}methylplatinum(II)}

\section{Zi-Jia Wang, Xiao-Xi Wang and Chong-Qing Wan}

\section{S1. Comment}

The hemilabile tridentate ligands with $\operatorname{PXP}(\mathrm{X}=\mathrm{C}, \mathrm{N}, \mathrm{O}, \mathrm{S}$, and As) donor sets have attracted particular attention for their coordinative extensibility and structural diversity with transition metals (Ainscough et al., 2004; Song, et al., 2002; Kunz et al., 2010; Wang, et al., 2010), as well as the catalytic property of their metal complexes (Zhang et al., 1996).

In the present context, we report a new complex of 2,6-bis(N-benzyl-N-diphenylphosphinomethylamino)pyridine ligand (abbreviated as L), namely $\mathrm{Pt}(\mathrm{L}) \mathrm{MeCl}$, (I). In the title complex, two phosphine atoms of the pendant diphenylphosinomethylamino arms bond to the square-planar coordinated $\mathrm{P}^{\mathrm{II}}$ ion at the $c i s$ sites, generating a 10-membered ring similar to that of the reported $\mathrm{Pt}(\mathrm{L}) \mathrm{Cl}_{2} \cdot \mathrm{CH}_{2} \mathrm{Cl}_{2}$ ( $\mathrm{Li}$ et al., 2005), as shown in Fig. 1. The mononuclear units are arranged along the c direction and interconnect through $\mathrm{C}-\mathrm{H}$ (methylene).. $\pi$ and $\mathrm{C}-\mathrm{H}$ (benzene) $\cdots \pi$ interactions (Umezawa et al., 1998) to

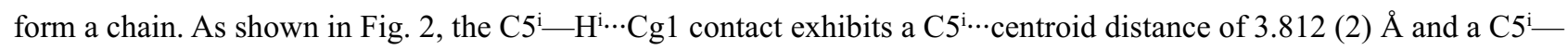
$\mathrm{H}^{\mathrm{i} \cdots}$ centroid angle of $146^{\circ}$, while the $\mathrm{C} 26^{\mathrm{ii}}-\mathrm{H}$ (methylene) interaction has a $\mathrm{C} 26^{\mathrm{ii} \cdots}$ centroid separation of 3.807 (2) $\AA$ and a $\mathrm{C} 26^{\mathrm{ii}}-\mathrm{H}^{\mathrm{i} \ldots} \cdots$ centroid angle of $147^{\circ}[\mathrm{Cg} 1$ presents ring of $\mathrm{C} 15-\mathrm{C} 20$ (benzene), symmetry codes: $\mathrm{i}-x+1.5,-y+1, z+0.5$; ii $-x+1.5,-y+1, z-0.5]$.

\section{S2. Experimental}

The 2,6-bis(N-benzyl-N-diphenylphosphinomethylamino)pyridine (L) was synthesized through the procedure as the literature (Li et al., 2005). The solid of $\mathrm{Pt}(\mathrm{COD}) \mathrm{MeCl}(0.210 \mathrm{~g}, 0.60 \mathrm{mmol})$ was added to the solution of $\mathrm{L}(0.411 \mathrm{~g}, 0.6$ $\mathrm{mmol})$ in $\mathrm{CH}_{2} \mathrm{Cl}_{2}(30 \mathrm{ml})$ and the mixture was stirred for one hour at room temperature. The yellow solvent was removed under vacuum and the title complex was obtained as a yellow powder, the block like crystals of which were obtained after four days by recrystallization from $\mathrm{CH}_{2} \mathrm{Cl}_{2} / \mathrm{n}$-hexane $(0.447 \mathrm{~g}, 80 \%$ yield).

\section{S3. Refinement}

The hydrogen atoms were placed in idealized positions and allowed to ride on the relevant carbon atoms, with $\mathrm{C}-\mathrm{H}=$ 0.93, 0.97 and $0.96 \AA$ for aryl, methylene and methyl hydrogens, respectively. $U_{i s o}(\mathrm{H})=1.2 U_{e q}(\mathrm{C})$. 


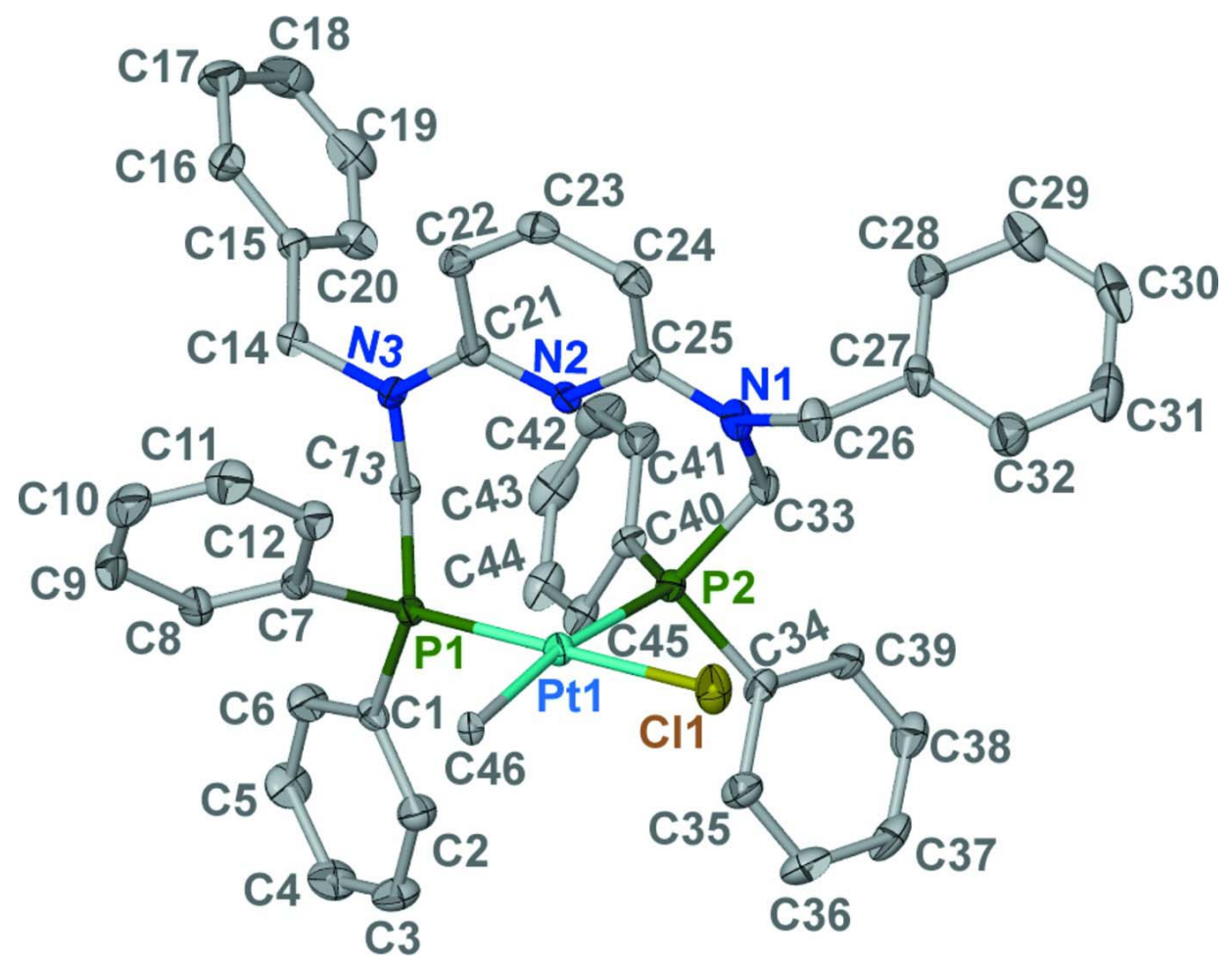

\section{Figure 1}

The atom-numbering scheme of the title complex I.Displacement ellipsoids are drawn at the $30 \%$ probability level and $\mathrm{H}$ atoms are omitted for clarity.

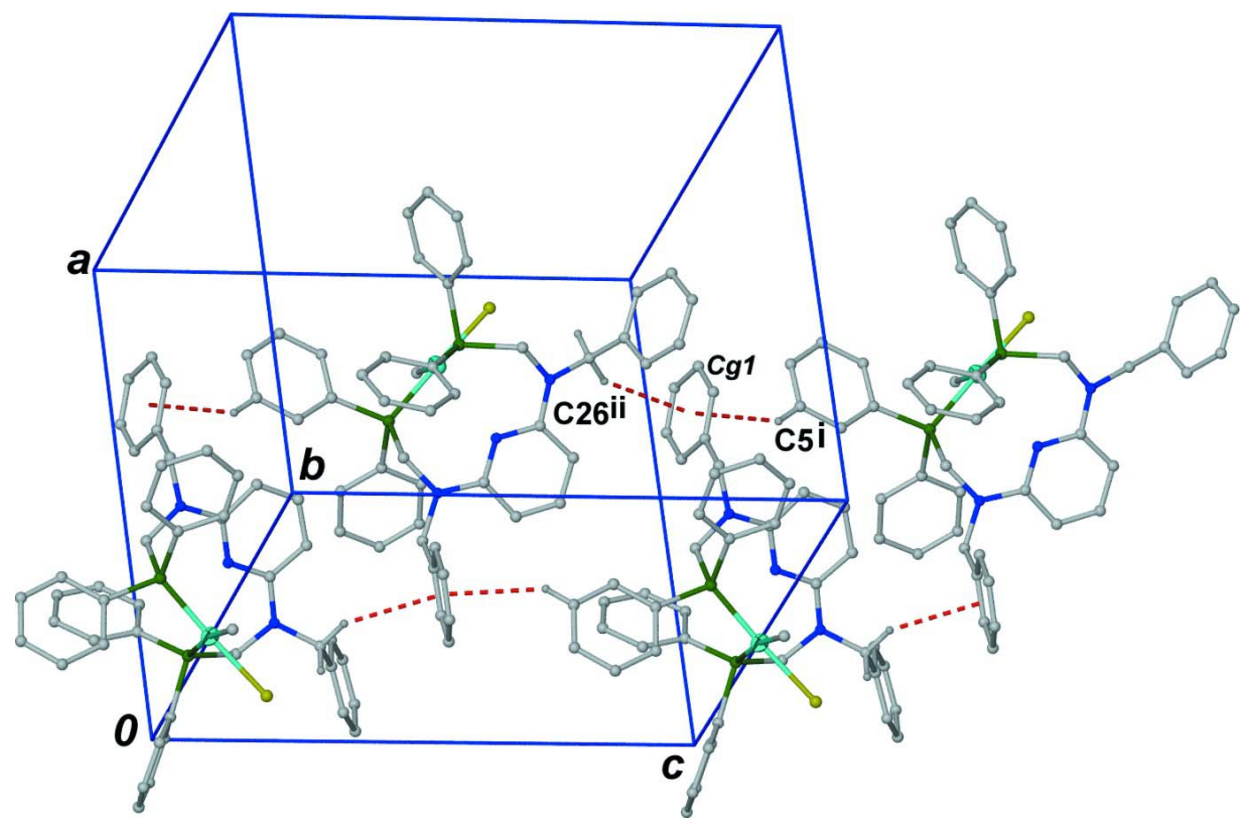

Figure 2

The $\mathrm{C}-\mathrm{H}$ (benzene) $\cdots \pi$ interactions between the mononuclear $\mathrm{Pt}(\mathrm{L}) \mathrm{MeCl}$ units arranged along the $c$ direction. The reddashed lines indicate the $\mathrm{C}-\mathrm{H} \cdots \pi$ interactions, while the $\mathrm{Cg} 1$ presents ring of $\mathrm{C} 15-\mathrm{C} 20$ (benzene). symmetry codes: $\mathrm{i}-x$ $+1.5,-y+1, z+0.5 ;$ ii $-x+1.5,-y+1, z-0.5$ 


\section{Chlorido $\left\{N^{2}, N^{6}\right.$-dibenzyl- $N^{2}, N^{6}$-bis[(diphenylphosphanyl)methyl]pyridine-2,6-diamine $\}$ methylplatinum(II)}

Crystal data

$\left[\mathrm{Pt}\left(\mathrm{CH}_{3}\right) \mathrm{Cl}\left(\mathrm{C}_{45} \mathrm{H}_{41} \mathrm{~N}_{3} \mathrm{P}_{2}\right)\right]$

$M_{r}=931.32$

Orthorhombic, $P 2_{1} 2_{1} 2_{1}$

Hall symbol: P 2ac 2ab

$a=15.3515$ (9) $\AA$

$b=15.9294(10) \AA$

$c=16.5197(10) \AA$

$V=4039.7(4) \AA^{3}$

\section{Data collection}

Bruker APEXII CCD area-detector diffractometer

Radiation source: fine-focus sealed tube Graphite monochromator

$\omega$ scans

Absorption correction: multi-scan

(SADABS; Bruker, 2007)

$T_{\text {min }}=0.640, T_{\max }=1.000$

\section{Refinement}

Refinement on $F^{2}$

Least-squares matrix: full

$R\left[F^{2}>2 \sigma\left(F^{2}\right)\right]=0.024$

$w R\left(F^{2}\right)=0.050$

$S=0.97$

7108 reflections

479 parameters

0 restraints

Primary atom site location: structure-invariant direct methods

\author{
$Z=4$ \\ $F(000)=1864$ \\ $D_{\mathrm{x}}=1.531 \mathrm{Mg} \mathrm{m}^{-3}$ \\ Mo $K \alpha$ radiation, $\lambda=0.71073 \AA$ \\ $\mu=3.66 \mathrm{~mm}^{-1}$ \\ $T=293 \mathrm{~K}$ \\ Block, colorless \\ $0.32 \times 0.24 \times 0.16 \mathrm{~mm}$
}

21886 measured reflections 7108 independent reflections 6498 reflections with $I>2 \sigma(I)$

$R_{\text {int }}=0.029$

$\theta_{\text {max }}=25.0^{\circ}, \theta_{\min }=1.8^{\circ}$

$h=-15 \rightarrow 18$

$k=-18 \rightarrow 18$

$l=-17 \rightarrow 19$

Secondary atom site location: difference Fourier map

Hydrogen site location: inferred from

neighbouring sites

$\mathrm{H}$-atom parameters constrained

$w=1 /\left[\sigma^{2}\left(F_{\mathrm{o}}^{2}\right)+(0 . P)^{2}\right] P=\left(F_{\mathrm{o}}^{2}+2 F_{\mathrm{c}}^{2}\right) / 3$

$(\Delta / \sigma)_{\max }=0.003$

$\Delta \rho_{\max }=0.74$ e $\AA^{-3}$

$\Delta \rho_{\min }=-0.38$ e $\AA^{-3}$

Absolute structure: Flack (1983), 3125 Friedel

pairs

Absolute structure parameter: -0.010 (4)

\section{Special details}

Geometry. All esds (except the esd in the dihedral angle between two 1.s. planes) are estimated using the full covariance matrix. The cell esds are taken into account individually in the estimation of esds in distances, angles and torsion angles; correlations between esds in cell parameters are only used when they are defined by crystal symmetry. An approximate (isotropic) treatment of cell esds is used for estimating esds involving l.s. planes.

Refinement. Refinement of $\mathrm{F}^{2}$ against ALL reflections. The weighted R-factor wR and goodness of fit $\mathrm{S}$ are based on $\mathrm{F}^{2}$, conventional R-factors $R$ are based on $F$, with $F$ set to zero for negative $F^{2}$. The threshold expression of $F^{2}>2 \operatorname{sigma}\left(\mathrm{F}^{2}\right)$ is used only for calculating R-factors(gt) etc. and is not relevant to the choice of reflections for refinement. R-factors based on $\mathrm{F}^{2}$ are statistically about twice as large as those based on F, and R- factors based on ALL data will be even larger.

Fractional atomic coordinates and isotropic or equivalent isotropic displacement parameters $\left(\AA^{2}\right)$

\begin{tabular}{lllll}
\hline & $x$ & $y$ & $z$ & $U_{\text {iso }} * U_{\text {eq }}$ \\
\hline Pt1 & $0.442088(10)$ & $0.536151(10)$ & $0.964197(10)$ & $0.03462(5)$ \\
C11 & $0.33715(9)$ & $0.50166(8)$ & $1.06371(8)$ & $0.0633(4)$ \\
P1 & $0.54103(7)$ & $0.57645(7)$ & $0.87509(7)$ & $0.0328(3)$ \\
P2 & $0.47428(6)$ & $0.39415(6)$ & $0.96383(8)$ & $0.0344(2)$
\end{tabular}




\begin{tabular}{|c|c|c|c|c|}
\hline N1 & $0.5395(2)$ & $0.4046(2)$ & $1.1248(2)$ & $0.0444(9)$ \\
\hline N2 & $0.62990(18)$ & $0.47138(19)$ & $1.03402(19)$ & $0.0340(7)$ \\
\hline N3 & $0.7081(2)$ & $0.5456(2)$ & $0.93740(19)$ & $0.0386(9)$ \\
\hline $\mathrm{C} 1$ & $0.4996(3)$ & $0.5651(2)$ & $0.7722(3)$ & $0.0371(10)$ \\
\hline $\mathrm{C} 2$ & $0.4096(3)$ & $0.5615(3)$ & $0.7627(3)$ & $0.0513(13)$ \\
\hline $\mathrm{H} 2$ & 0.3739 & 0.5667 & 0.8079 & $0.062 *$ \\
\hline $\mathrm{C} 3$ & $0.3728(3)$ & 0.5505 (4) & 0.6874 (3) & $0.0627(15)$ \\
\hline H3 & 0.3125 & 0.5483 & 0.6821 & $0.075^{*}$ \\
\hline C4 & $0.4240(4)$ & $0.5428(4)$ & $0.6211(3)$ & $0.0686(14)$ \\
\hline H4 & 0.3990 & 0.5348 & 0.5704 & $0.082 *$ \\
\hline $\mathrm{C} 5$ & $0.5120(4)$ & $0.5466(4)$ & $0.6290(3)$ & $0.0693(15)$ \\
\hline H5 & 0.5469 & 0.5418 & 0.5832 & $0.083^{*}$ \\
\hline $\mathrm{C} 6$ & 0.5504 (3) & $0.5576(3)$ & 0.7034 (3) & $0.0568(13)$ \\
\hline H6 & 0.6108 & 0.5600 & 0.7075 & $0.068^{*}$ \\
\hline $\mathrm{C} 7$ & $0.5844(3)$ & 0.6819 (2) & 0.8848 (3) & $0.0397(10)$ \\
\hline $\mathrm{C} 8$ & $0.6100(3)$ & $0.7296(3)$ & 0.8197 (3) & $0.0525(12)$ \\
\hline H8 & 0.5969 & 0.7123 & 0.7674 & $0.063^{*}$ \\
\hline C9 & $0.6558(4)$ & 0.8044 (3) & 0.8325 (4) & $0.0717(17)$ \\
\hline H9 & 0.6739 & 0.8365 & 0.7886 & $0.086^{*}$ \\
\hline $\mathrm{C} 10$ & $0.6739(4)$ & 0.8304 (3) & $0.9096(5)$ & 0.0745 (18) \\
\hline H10 & 0.7040 & 0.8803 & 0.9180 & $0.089^{*}$ \\
\hline C11 & $0.6482(3)$ & $0.7836(3)$ & 0.9737 (4) & 0.0709 (16) \\
\hline H11 & 0.6613 & 0.8015 & 1.0259 & $0.085^{*}$ \\
\hline $\mathrm{C} 12$ & $0.6028(3)$ & $0.7101(3)$ & 0.9627 (3) & $0.0506(11)$ \\
\hline H12 & 0.5844 & 0.6791 & 1.0072 & $0.061^{*}$ \\
\hline $\mathrm{C} 13$ & $0.6476(2)$ & 0.5207 (3) & 0.8754 (2) & $0.0387(10)$ \\
\hline H13A & 0.6366 & 0.4610 & 0.8811 & $0.046^{*}$ \\
\hline H13B & 0.6751 & 0.5291 & 0.8231 & $0.046^{*}$ \\
\hline C14 & $0.7841(3)$ & $0.5946(3)$ & $0.9142(3)$ & $0.0430(11)$ \\
\hline $\mathrm{H} 14 \mathrm{~A}$ & 0.7908 & 0.6408 & 0.9519 & $0.052 *$ \\
\hline H14B & 0.7742 & 0.6184 & 0.8609 & $0.052 *$ \\
\hline C15 & $0.8671(3)$ & $0.5450(3)$ & 0.9125 (2) & $0.0427(10)$ \\
\hline C16 & $0.9462(3)$ & 0.5834 (3) & $0.9267(3)$ & $0.0582(12)$ \\
\hline H16 & 0.9473 & 0.6399 & 0.9408 & $0.070^{*}$ \\
\hline $\mathrm{C} 17$ & $1.0234(3)$ & $0.5404(5)$ & 0.9205 (3) & $0.0762(16)$ \\
\hline H17 & 1.0761 & 0.5674 & 0.9303 & $0.091^{*}$ \\
\hline $\mathrm{C} 18$ & $1.0216(4)$ & $0.4565(6)$ & $0.8994(4)$ & 0.087 (2) \\
\hline H18 & 1.0736 & 0.4269 & 0.8943 & $0.104 *$ \\
\hline C19 & $0.9465(5)$ & $0.4176(4)$ & $0.8865(3)$ & $0.0764(17)$ \\
\hline H19 & 0.9461 & 0.3608 & 0.8735 & $0.092 *$ \\
\hline $\mathrm{C} 20$ & 0.8677 (3) & 0.4615 (4) & $0.8924(3)$ & $0.0580(12)$ \\
\hline $\mathrm{H} 20$ & 0.8154 & 0.4337 & 0.8827 & $0.070 *$ \\
\hline $\mathrm{C} 21$ & 0.6965 (2) & $0.5226(2)$ & $1.0174(2)$ & $0.0369(10)$ \\
\hline $\mathrm{C} 22$ & $0.7514(3)$ & 0.5538 (3) & $1.0782(3)$ & $0.0425(11)$ \\
\hline H22 & 0.7995 & 0.5868 & 1.0656 & $0.051^{*}$ \\
\hline $\mathrm{C} 23$ & $0.7319(3)$ & $0.5341(3)$ & $1.1561(3)$ & $0.0480(11)$ \\
\hline $\mathrm{H} 23$ & 0.7677 & 0.5538 & 1.1974 & $0.058^{*}$ \\
\hline C24 & $0.6610(3)$ & $0.4863(3)$ & $1.1754(3)$ & $0.0462(12)$ \\
\hline
\end{tabular}




\begin{tabular}{|c|c|c|c|c|}
\hline $\mathrm{H} 24$ & 0.6466 & 0.4752 & 1.2290 & $0.055^{*}$ \\
\hline $\mathrm{C} 25$ & 0.6110 (3) & 0.4545 (3) & 1.1117 (2) & $0.0370(9)$ \\
\hline $\mathrm{C} 26$ & $0.4923(3)$ & $0.4046(3)$ & $1.2028(3)$ & $0.0496(12)$ \\
\hline $\mathrm{H} 26 \mathrm{~A}$ & 0.4324 & 0.4212 & 1.1933 & $0.060^{*}$ \\
\hline H26B & 0.5185 & 0.4461 & 1.2382 & $0.060^{*}$ \\
\hline $\mathrm{C} 27$ & $0.4930(3)$ & 0.3215 (3) & $1.2446(3)$ & $0.0458(12)$ \\
\hline $\mathrm{C} 28$ & $0.5690(4)$ & 0.2808 (3) & $1.2634(3)$ & $0.0626(14)$ \\
\hline $\mathrm{H} 28$ & 0.6221 & 0.3046 & 1.2489 & $0.075^{*}$ \\
\hline $\mathrm{C} 29$ & $0.5676(5)$ & 0.2044 (4) & $1.3036(3)$ & $0.0746(16)$ \\
\hline H29 & 0.6199 & 0.1774 & 1.3151 & $0.089^{*}$ \\
\hline $\mathrm{C} 30$ & $0.4926(6)$ & 0.1689 (4) & $1.3263(4)$ & $0.086(2)$ \\
\hline H30 & 0.4927 & 0.1178 & 1.3535 & $0.104 *$ \\
\hline C31 & $0.4153(5)$ & 0.2087 (4) & 1.3089 (4) & $0.090(2)$ \\
\hline H31 & 0.3628 & 0.1847 & 1.3249 & $0.108^{*}$ \\
\hline $\mathrm{C} 32$ & $0.4153(4)$ & $0.2852(3)$ & $1.2672(3)$ & $0.0660(15)$ \\
\hline H32 & 0.3629 & 0.3115 & 1.2548 & $0.079 *$ \\
\hline C33 & $0.5016(3)$ & 0.3507 (3) & $1.0645(3)$ & $0.0434(11)$ \\
\hline $\mathrm{H} 33 \mathrm{~A}$ & 0.4486 & 0.3274 & 1.0872 & $0.052^{*}$ \\
\hline H33B & 0.5414 & 0.3042 & 1.0561 & $0.052 *$ \\
\hline C34 & $0.3790(3)$ & 0.3301 (3) & $0.9376(3)$ & $0.0412(11)$ \\
\hline C35 & $0.3074(3)$ & 0.3654 (3) & $0.9016(3)$ & $0.0549(13)$ \\
\hline H35 & 0.3056 & 0.4232 & 0.8936 & $0.066^{*}$ \\
\hline $\mathrm{C} 36$ & $0.2383(3)$ & $0.3169(4)$ & $0.8772(4)$ & $0.0724(16)$ \\
\hline H36 & 0.1905 & 0.3414 & 0.8521 & $0.087^{*}$ \\
\hline C37 & $0.2406(4)$ & 0.2315 (4) & 0.8903 (4) & $0.0698(16)$ \\
\hline H37 & 0.1936 & 0.1984 & 0.8747 & $0.084 *$ \\
\hline $\mathrm{C} 38$ & 0.3104 (4) & 0.1956 (3) & 0.9257 (3) & $0.0635(15)$ \\
\hline H38 & 0.3116 & 0.1379 & 0.9344 & $0.076^{*}$ \\
\hline C39 & 0.3789 (3) & 0.2437 (3) & 0.9485 (3) & $0.0508(12)$ \\
\hline H39 & 0.4271 & 0.2182 & 0.9722 & $0.061 *$ \\
\hline $\mathrm{C} 40$ & $0.5494(3)$ & 0.3437 (2) & $0.8929(3)$ & $0.0371(10)$ \\
\hline $\mathrm{C} 41$ & 0.5277 (3) & $0.3486(3)$ & $0.8112(3)$ & $0.0501(12)$ \\
\hline H41 & 0.4761 & 0.3743 & 0.7952 & $0.060 *$ \\
\hline $\mathrm{C} 42$ & $0.5840(4)$ & 0.3148 (3) & $0.7537(3)$ & $0.0637(15)$ \\
\hline $\mathrm{H} 42$ & 0.5696 & 0.3183 & 0.6991 & $0.076^{*}$ \\
\hline $\mathrm{C} 43$ & 0.6587 (4) & $0.2773(4)$ & $0.7757(5)$ & 0.0791 (19) \\
\hline H43 & 0.6955 & 0.2553 & 0.7364 & $0.095^{*}$ \\
\hline $\mathrm{C} 44$ & $0.6806(4)$ & $0.2714(3)$ & $0.8551(5)$ & $0.0746(18)$ \\
\hline H44 & 0.7323 & 0.2450 & 0.8700 & $0.089^{*}$ \\
\hline $\mathrm{C} 45$ & $0.6265(3)$ & 0.3044 (3) & $0.9140(3)$ & $0.0541(13)$ \\
\hline H45 & 0.6420 & 0.3002 & 0.9683 & $0.065^{*}$ \\
\hline C46 & $0.3910(2)$ & 0.66327 (19) & $0.9668(3)$ & $0.0264(8)$ \\
\hline H46A & 0.4009 & 0.6874 & 1.0193 & $0.040 *$ \\
\hline H46B & 0.3296 & 0.6621 & 0.9558 & $0.040 *$ \\
\hline $\mathrm{H} 46 \mathrm{C}$ & 0.4199 & 0.6965 & 0.9264 & $0.040^{*}$ \\
\hline
\end{tabular}


Atomic displacement parameters $\left(\AA^{2}\right)$

\begin{tabular}{|c|c|c|c|c|c|c|}
\hline & $U^{11}$ & $U^{22}$ & $U^{33}$ & $U^{12}$ & $U^{13}$ & $U^{23}$ \\
\hline Pt1 & $0.03517(8)$ & $0.03335(7)$ & $0.03532(8)$ & $0.00224(8)$ & $0.00569(8)$ & $0.00014(9)$ \\
\hline $\mathrm{Cl1}$ & $0.0630(8)$ & $0.0570(6)$ & $0.0698(9)$ & $0.0094(6)$ & $0.0314(7)$ & $0.0093(6)$ \\
\hline P1 & $0.0343(7)$ & 0.0330 & $0.0310(6)$ & -0.0013 & $0.0000(5)$ & $0.0017(5)$ \\
\hline $\mathrm{P} 2$ & $0.0359(5)$ & $0.0318(5)$ & $0.0354(6)$ & $-0.0028(4)$ & $0.0006(6)$ & $-0.0013(6)$ \\
\hline N1 & $0.063(3)$ & $0.044(2)$ & 0.0255 (19) & $-0.0086(18)$ & 0.0047 (17) & $-0.0020(16)$ \\
\hline $\mathrm{N} 2$ & 0.0392 (17) & $0.0321(16)$ & $0.0306(17)$ & $-0.0007(15)$ & $-0.0022(16)$ & $0.002(2)$ \\
\hline N3 & $0.0324(18)$ & $0.046(2)$ & $0.037(2)$ & $-0.0084(17)$ & 0.0007 (14) & $0.0068(18)$ \\
\hline $\mathrm{C} 1$ & 0.048 & $0.033(2)$ & $0.031(2)$ & -0.0003 (19) & $-0.005(2)$ & 0.0005 (18) \\
\hline $\mathrm{C} 2$ & $0.047(3)$ & $0.058(3)$ & $0.049(3)$ & $-0.001(2)$ & $-0.001(2)$ & $-0.001(2)$ \\
\hline C3 & $0.048(3)$ & $0.080(4)$ & $0.060(3)$ & -0.005 & $-0.016(3)$ & -0.011 \\
\hline $\mathrm{C} 4$ & $0.078(4)$ & $0.084(4)$ & $0.044(3)$ & $-0.002(3)$ & $-0.016(3)$ & -0.007 (3) \\
\hline $\mathrm{C} 5$ & $0.080(4)$ & $0.095(4)$ & $0.033(3)$ & $-0.006(4)$ & $0.002(3)$ & -0.008 \\
\hline C6 & $0.053(3)$ & $0.080(4)$ & $0.037(3)$ & -0.003 & $0.001(3)$ & $0.002(2)$ \\
\hline $\mathrm{C} 7$ & $0.038(3)$ & $0.033(2)$ & $0.047(3)$ & $-0.0015(18)$ & $0.001(2)$ & $-0.001(2)$ \\
\hline $\mathrm{C} 8$ & $0.057(3)$ & $0.046(3)$ & $0.054(3)$ & $-0.006(2)$ & -0.004 & $0.008(2)$ \\
\hline $\mathrm{C} 9$ & $0.066(4)$ & 0.047 (3) & $0.102(5)$ & $-0.012(3)$ & $0.002(4)$ & $0.027(3)$ \\
\hline $\mathrm{C} 10$ & $0.063(4)$ & $0.044(3)$ & $0.117(6)$ & $-0.016(3)$ & $-0.016(4)$ & $-0.006(3)$ \\
\hline C11 & $0.076(4)$ & $0.051(3)$ & $0.085(4)$ & -0.005 & $-0.016(4)$ & $-0.017(3)$ \\
\hline $\mathrm{C} 12$ & $0.054(3)$ & $0.042(2)$ & $0.055(3)$ & $-0.002(2)$ & $-0.002(3)$ & -0.007 \\
\hline $\mathrm{C} 13$ & $0.040(2)$ & $0.041(3)$ & $0.036(2)$ & $-0.0030(19)$ & 0.0029 (19) & $0.005(2)$ \\
\hline $\mathrm{C} 14$ & $0.039(2)$ & $0.044(3)$ & $0.046(3)$ & $-0.005(2)$ & $0.000(2)$ & 0.007 (2) \\
\hline C15 & $0.041(2)$ & $0.056(3)$ & $0.032(2)$ & $-0.003(2)$ & $0.0002(18)$ & 0.009 (2) \\
\hline $\mathrm{C} 16$ & $0.048(3)$ & $0.073(3)$ & $0.053(3)$ & $-0.013(3)$ & -0.001 & $0.011(2)$ \\
\hline $\mathrm{C} 17$ & $0.042(3)$ & $0.115(5)$ & $0.072(4)$ & 0.007 (4) & $0.001(3)$ & $0.018(4)$ \\
\hline $\mathrm{C} 18$ & $0.061(4)$ & $0.135(7)$ & $0.065(4)$ & $0.044(5)$ & $0.016(3)$ & $0.018(5)$ \\
\hline C19 & $0.096(5)$ & $0.080(4)$ & $0.054(3)$ & $0.035(4)$ & $0.016(4)$ & $0.004(3)$ \\
\hline $\mathrm{C} 20$ & 0.065 & $0.060(3)$ & $0.049(3)$ & $0.006(3)$ & $-0.004(2)$ & $0.002(3)$ \\
\hline $\mathrm{C} 21$ & $0.038(2)$ & $0.029(2)$ & $0.044(3)$ & $0.0050(18)$ & $-0.0004(18)$ & $-0.0015(19)$ \\
\hline $\mathrm{C} 22$ & $0.040(2)$ & $0.041(3)$ & $0.046(3)$ & $-0.0045(19)$ & $-0.008(2)$ & $-0.004(2)$ \\
\hline $\mathrm{C} 23$ & $0.051(3)$ & $0.046(2)$ & $0.048(3)$ & $0.005(3)$ & $-0.015(2)$ & -0.008 \\
\hline $\mathrm{C} 24$ & $0.062(3)$ & $0.045(3)$ & $0.032(2)$ & $0.004(2)$ & $-0.007(2)$ & $-0.003(2)$ \\
\hline $\mathrm{C} 25$ & $0.045(2)$ & $0.030(2)$ & $0.035(2)$ & $0.003(2)$ & $-0.0004(19)$ & $-0.001(2)$ \\
\hline $\mathrm{C} 26$ & $0.062(3)$ & $0.051(3)$ & $0.036(3)$ & $-0.002(2)$ & $0.013(2)$ & $0.001(2)$ \\
\hline $\mathrm{C} 27$ & $0.062(3)$ & $0.048(3)$ & $0.027(2)$ & $-0.012(2)$ & $0.008(2)$ & $0.002(2)$ \\
\hline $\mathrm{C} 28$ & $0.069(4)$ & $0.065(3)$ & $0.054(3)$ & $0.000(3)$ & $0.008(3)$ & $0.005(3)$ \\
\hline $\mathrm{C} 29$ & $0.104(5)$ & $0.070(4)$ & $0.049(3)$ & $0.013(4)$ & $0.003(4)$ & $0.011(3)$ \\
\hline $\mathrm{C} 30$ & $0.148(7)$ & $0.061(4)$ & $0.051(4)$ & $-0.014(4)$ & $0.000(4)$ & $0.019(3)$ \\
\hline C31 & $0.107(6)$ & $0.079(4)$ & $0.084(5)$ & $-0.041(4)$ & $0.015(4)$ & $0.018(4)$ \\
\hline $\mathrm{C} 32$ & $0.070(4)$ & $0.073(4)$ & $0.054(3)$ & $-0.014(3)$ & $-0.002(3)$ & 0.007 (3) \\
\hline $\mathrm{C} 33$ & $0.053(3)$ & $0.037(2)$ & $0.040(3)$ & $-0.012(2)$ & $-0.001(2)$ & $0.004(2)$ \\
\hline C34 & $0.034(2)$ & $0.048(3)$ & $0.042(3)$ & $-0.004(2)$ & $0.008(2)$ & $-0.006(2)$ \\
\hline $\mathrm{C} 35$ & $0.043(3)$ & $0.050(3)$ & $0.071(4)$ & $-0.003(2)$ & $-0.006(3)$ & $-0.006(3)$ \\
\hline $\mathrm{C} 36$ & $0.039(3)$ & $0.085(4)$ & $0.094(5)$ & $-0.004(3)$ & $-0.008(3)$ & $-0.014(4)$ \\
\hline C37 & $0.055(4)$ & $0.078(4)$ & 0.077 (4) & $-0.034(3)$ & $0.008(3)$ & -0.020 \\
\hline C38 & $0.070(4)$ & $0.052(3)$ & $0.068(4)$ & $-0.022(3)$ & $0.002(3)$ & $-0.008(3)$ \\
\hline
\end{tabular}


supporting information

\begin{tabular}{lllllll} 
C39 & $0.046(3)$ & $0.041(2)$ & $0.066(4)$ & $-0.009(2)$ & $-0.009(2)$ & $0.001(2)$ \\
C40 & $0.034(2)$ & $0.034(2)$ & $0.043(3)$ & $-0.001(2)$ & $0.000(2)$ & $-0.0075(19)$ \\
C41 & $0.048(3)$ & $0.050(3)$ & $0.052(3)$ & $-0.014(2)$ & $0.008(2)$ & $-0.010(2)$ \\
C42 & $0.066(4)$ & $0.070(3)$ & $0.055(3)$ & $-0.020(3)$ & $0.016(3)$ & $-0.022(3)$ \\
C43 & $0.066(4)$ & $0.071(4)$ & $0.100(5)$ & $-0.009(3)$ & $0.030(4)$ & $-0.039(4)$ \\
C44 & $0.050(3)$ & $0.061(4)$ & $0.112(6)$ & $0.013(3)$ & $0.012(4)$ & $-0.019(4)$ \\
C45 & $0.048(3)$ & $0.049(3)$ & $0.065(3)$ & $0.003(2)$ & $-0.001(3)$ & $-0.004(3)$ \\
C46 & $0.0311(19)$ & $0.0184(16)$ & $0.030(2)$ & $0.0069(14)$ & $0.011(2)$ & $-0.0014(18)$ \\
\hline
\end{tabular}

Geometric parameters $\left(\AA,{ }^{\circ}\right)$

\begin{tabular}{|c|c|c|c|}
\hline $\mathrm{Pt} 1-\mathrm{C} 46$ & $2.172(3)$ & C18-H18 & 0.9300 \\
\hline $\mathrm{Pt} 1-\mathrm{P} 1$ & $2.2105(11)$ & $\mathrm{C} 19-\mathrm{C} 20$ & $1.400(7)$ \\
\hline $\mathrm{Pt} 1-\mathrm{P} 2$ & $2.3153(10)$ & C19-H19 & 0.9300 \\
\hline $\mathrm{Pt} 1-\mathrm{Cl1}$ & $2.3663(12)$ & $\mathrm{C} 20-\mathrm{H} 20$ & 0.9300 \\
\hline $\mathrm{P} 1-\mathrm{C} 7$ & $1.814(4)$ & $\mathrm{C} 21-\mathrm{C} 22$ & $1.402(6)$ \\
\hline $\mathrm{P} 1-\mathrm{C} 1$ & $1.824(4)$ & $\mathrm{C} 22-\mathrm{C} 23$ & $1.358(6)$ \\
\hline $\mathrm{P} 1-\mathrm{C} 13$ & $1.862(4)$ & $\mathrm{C} 22-\mathrm{H} 22$ & 0.9300 \\
\hline $\mathrm{P} 2-\mathrm{C} 40$ & $1.830(4)$ & $\mathrm{C} 23-\mathrm{C} 24$ & $1.366(6)$ \\
\hline $\mathrm{P} 2-\mathrm{C} 34$ & $1.836(4)$ & $\mathrm{C} 23-\mathrm{H} 23$ & 0.9300 \\
\hline $\mathrm{P} 2-\mathrm{C} 33$ & $1.850(4)$ & $\mathrm{C} 24-\mathrm{C} 25$ & $1.397(6)$ \\
\hline $\mathrm{N} 1-\mathrm{C} 25$ & $1.373(5)$ & $\mathrm{C} 24-\mathrm{H} 24$ & 0.9300 \\
\hline $\mathrm{N} 1-\mathrm{C} 33$ & $1.438(5)$ & $\mathrm{C} 26-\mathrm{C} 27$ & $1.493(6)$ \\
\hline $\mathrm{N} 1-\mathrm{C} 26$ & $1.478(5)$ & $\mathrm{C} 26-\mathrm{H} 26 \mathrm{~A}$ & 0.9700 \\
\hline $\mathrm{N} 2-\mathrm{C} 21$ & $1.336(5)$ & C26-H26B & 0.9700 \\
\hline $\mathrm{N} 2-\mathrm{C} 25$ & $1.343(5)$ & $\mathrm{C} 27-\mathrm{C} 28$ & $1.370(7)$ \\
\hline $\mathrm{N} 3-\mathrm{C} 21$ & $1.383(5)$ & $\mathrm{C} 27-\mathrm{C} 32$ & $1.377(7)$ \\
\hline $\mathrm{N} 3-\mathrm{C} 13$ & $1.439(5)$ & $\mathrm{C} 28-\mathrm{C} 29$ & $1.386(7)$ \\
\hline $\mathrm{N} 3-\mathrm{C} 14$ & $1.455(5)$ & $\mathrm{C} 28-\mathrm{H} 28$ & 0.9300 \\
\hline $\mathrm{C} 1-\mathrm{C} 6$ & $1.384(6)$ & $\mathrm{C} 29-\mathrm{C} 30$ & $1.336(9)$ \\
\hline $\mathrm{C} 1-\mathrm{C} 2$ & $1.390(6)$ & $\mathrm{C} 29-\mathrm{H} 29$ & 0.9300 \\
\hline $\mathrm{C} 2-\mathrm{C} 3$ & $1.378(6)$ & $\mathrm{C} 30-\mathrm{C} 31$ & $1.377(9)$ \\
\hline $\mathrm{C} 2-\mathrm{H} 2$ & 0.9300 & $\mathrm{C} 30-\mathrm{H} 30$ & 0.9300 \\
\hline $\mathrm{C} 3-\mathrm{C} 4$ & $1.354(7)$ & $\mathrm{C} 31-\mathrm{C} 32$ & $1.400(8)$ \\
\hline $\mathrm{C} 3-\mathrm{H} 3$ & 0.9300 & C $31-\mathrm{H} 31$ & 0.9300 \\
\hline $\mathrm{C} 4-\mathrm{C} 5$ & $1.359(7)$ & C32-H32 & 0.9300 \\
\hline $\mathrm{C} 4-\mathrm{H} 4$ & 0.9300 & $\mathrm{C} 33-\mathrm{H} 33 \mathrm{~A}$ & 0.9700 \\
\hline $\mathrm{C} 5-\mathrm{C} 6$ & $1.374(6)$ & $\mathrm{C} 33-\mathrm{H} 33 \mathrm{~B}$ & 0.9700 \\
\hline $\mathrm{C} 5-\mathrm{H} 5$ & 0.9300 & $\mathrm{C} 34-\mathrm{C} 35$ & $1.370(6)$ \\
\hline C6- $\mathrm{H} 6$ & 0.9300 & C34-C39 & $1.387(6)$ \\
\hline $\mathrm{C} 7-\mathrm{C} 8$ & $1.375(6)$ & $\mathrm{C} 35-\mathrm{C} 36$ & $1.373(7)$ \\
\hline $\mathrm{C} 7-\mathrm{C} 12$ & $1.390(7)$ & C35-H35 & 0.9300 \\
\hline $\mathrm{C} 8-\mathrm{C} 9$ & $1.400(7)$ & $\mathrm{C} 36-\mathrm{C} 37$ & $1.377(8)$ \\
\hline $\mathrm{C} 8-\mathrm{H} 8$ & 0.9300 & C36-H36 & 0.9300 \\
\hline C9- $\mathrm{C} 10$ & $1.368(8)$ & C $37-\mathrm{C} 38$ & $1.349(8)$ \\
\hline C9-H9 & 0.9300 & С $37-\mathrm{H} 37$ & 0.9300 \\
\hline $\mathrm{C} 10-\mathrm{C} 11$ & $1.353(8)$ & C38-C39 & $1.354(6)$ \\
\hline $\mathrm{C} 10-\mathrm{H} 10$ & 0.9300 & C38-H38 & 0.9300 \\
\hline
\end{tabular}




\begin{tabular}{|c|c|c|c|}
\hline $\mathrm{C} 11-\mathrm{C} 12$ & $1.376(6)$ & C39-H39 & 0.9300 \\
\hline C11-H11 & 0.9300 & $\mathrm{C} 40-\mathrm{C} 45$ & $1.384(6)$ \\
\hline $\mathrm{C} 12-\mathrm{H} 12$ & 0.9300 & $\mathrm{C} 40-\mathrm{C} 41$ & $1.392(6)$ \\
\hline C13-H13A & 0.9700 & $\mathrm{C} 41-\mathrm{C} 42$ & $1.393(7)$ \\
\hline C13-H13B & 0.9700 & $\mathrm{C} 41-\mathrm{H} 41$ & 0.9300 \\
\hline $\mathrm{C} 14-\mathrm{C} 15$ & $1.499(6)$ & $\mathrm{C} 42-\mathrm{C} 43$ & $1.343(8)$ \\
\hline C14-H14A & 0.9700 & $\mathrm{C} 42-\mathrm{H} 42$ & 0.9300 \\
\hline C14-H14B & 0.9700 & $\mathrm{C} 43-\mathrm{C} 44$ & $1.358(8)$ \\
\hline $\mathrm{C} 15-\mathrm{C} 20$ & $1.371(7)$ & $\mathrm{C} 43-\mathrm{H} 43$ & 0.9300 \\
\hline $\mathrm{C} 15-\mathrm{C} 16$ & $1.380(6)$ & $\mathrm{C} 44-\mathrm{C} 45$ & $1.384(7)$ \\
\hline $\mathrm{C} 16-\mathrm{C} 17$ & $1.371(7)$ & $\mathrm{C} 44-\mathrm{H} 44$ & 0.9300 \\
\hline $\mathrm{C} 16-\mathrm{H} 16$ & 0.9300 & $\mathrm{C} 45-\mathrm{H} 45$ & 0.9300 \\
\hline $\mathrm{C} 17-\mathrm{C} 18$ & $1.382(10)$ & $\mathrm{C} 46-\mathrm{H} 46 \mathrm{~A}$ & 0.9600 \\
\hline $\mathrm{C} 17-\mathrm{H} 17$ & 0.9300 & $\mathrm{C} 46-\mathrm{H} 46 \mathrm{~B}$ & 0.9600 \\
\hline $\mathrm{C} 18-\mathrm{C} 19$ & $1.327(9)$ & $\mathrm{C} 46-\mathrm{H} 46 \mathrm{C}$ & 0.9600 \\
\hline $\mathrm{C} 46-\mathrm{Pt} 1-\mathrm{P} 1$ & $89.45(10)$ & $\mathrm{C} 15-\mathrm{C} 20-\mathrm{H} 20$ & 119.8 \\
\hline $\mathrm{C} 46-\mathrm{Pt} 1-\mathrm{P} 2$ & $171.11(9)$ & $\mathrm{C} 19-\mathrm{C} 20-\mathrm{H} 20$ & 119.8 \\
\hline $\mathrm{P} 1-\mathrm{Pt} 1-\mathrm{P} 2$ & $97.77(4)$ & $\mathrm{N} 2-\mathrm{C} 21-\mathrm{N} 3$ & $117.2(4)$ \\
\hline $\mathrm{C} 46-\mathrm{Pt} 1-\mathrm{C} 11$ & $87.54(10)$ & $\mathrm{N} 2-\mathrm{C} 21-\mathrm{C} 22$ & $121.9(4)$ \\
\hline $\mathrm{P} 1-\mathrm{Pt} 1-\mathrm{C} 11$ & $176.26(4)$ & $\mathrm{N} 3-\mathrm{C} 21-\mathrm{C} 22$ & $120.8(4)$ \\
\hline $\mathrm{P} 2-\mathrm{Pt} 1-\mathrm{C} 11$ & $85.43(4)$ & $\mathrm{C} 23-\mathrm{C} 22-\mathrm{C} 21$ & $117.7(4)$ \\
\hline $\mathrm{C} 7-\mathrm{P} 1-\mathrm{C} 1$ & $107.6(2)$ & $\mathrm{C} 23-\mathrm{C} 22-\mathrm{H} 22$ & 121.2 \\
\hline $\mathrm{C} 7-\mathrm{P} 1-\mathrm{C} 13$ & $96.84(18)$ & $\mathrm{C} 21-\mathrm{C} 22-\mathrm{H} 22$ & 121.2 \\
\hline $\mathrm{C} 1-\mathrm{P} 1-\mathrm{C} 13$ & $105.16(19)$ & $\mathrm{C} 22-\mathrm{C} 23-\mathrm{C} 24$ & $121.7(4)$ \\
\hline $\mathrm{C} 7-\mathrm{P} 1-\mathrm{Pt} 1$ & $117.51(15)$ & $\mathrm{C} 22-\mathrm{C} 23-\mathrm{H} 23$ & 119.1 \\
\hline $\mathrm{C} 1-\mathrm{P} 1-\mathrm{Pt} 1$ & $110.60(15)$ & $\mathrm{C} 24-\mathrm{C} 23-\mathrm{H} 23$ & 119.1 \\
\hline $\mathrm{C} 13-\mathrm{P} 1-\mathrm{Pt} 1$ & $117.62(13)$ & $\mathrm{C} 23-\mathrm{C} 24-\mathrm{C} 25$ & $117.6(4)$ \\
\hline $\mathrm{C} 40-\mathrm{P} 2-\mathrm{C} 34$ & $96.12(19)$ & $\mathrm{C} 23-\mathrm{C} 24-\mathrm{H} 24$ & 121.2 \\
\hline $\mathrm{C} 40-\mathrm{P} 2-\mathrm{C} 33$ & $105.6(2)$ & $\mathrm{C} 25-\mathrm{C} 24-\mathrm{H} 24$ & 121.2 \\
\hline $\mathrm{C} 34-\mathrm{P} 2-\mathrm{C} 33$ & $100.7(2)$ & $\mathrm{N} 2-\mathrm{C} 25-\mathrm{N} 1$ & $116.1(3)$ \\
\hline $\mathrm{C} 40-\mathrm{P} 2-\mathrm{Pt} 1$ & $124.42(14)$ & $\mathrm{N} 2-\mathrm{C} 25-\mathrm{C} 24$ & $121.9(4)$ \\
\hline $\mathrm{C} 34-\mathrm{P} 2-\mathrm{Pt} 1$ & $111.95(14)$ & $\mathrm{N} 1-\mathrm{C} 25-\mathrm{C} 24$ & $122.0(4)$ \\
\hline $\mathrm{C} 33-\mathrm{P} 2-\mathrm{Pt} 1$ & $114.30(15)$ & $\mathrm{N} 1-\mathrm{C} 26-\mathrm{C} 27$ & $113.6(4)$ \\
\hline $\mathrm{C} 25-\mathrm{N} 1-\mathrm{C} 33$ & $124.0(3)$ & $\mathrm{N} 1-\mathrm{C} 26-\mathrm{H} 26 \mathrm{~A}$ & 108.9 \\
\hline $\mathrm{C} 25-\mathrm{N} 1-\mathrm{C} 26$ & $122.0(3)$ & $\mathrm{C} 27-\mathrm{C} 26-\mathrm{H} 26 \mathrm{~A}$ & 108.9 \\
\hline $\mathrm{C} 33-\mathrm{N} 1-\mathrm{C} 26$ & $114.0(4)$ & $\mathrm{N} 1-\mathrm{C} 26-\mathrm{H} 26 \mathrm{~B}$ & 108.9 \\
\hline $\mathrm{C} 21-\mathrm{N} 2-\mathrm{C} 25$ & $119.0(3)$ & $\mathrm{C} 27-\mathrm{C} 26-\mathrm{H} 26 \mathrm{~B}$ & 108.9 \\
\hline $\mathrm{C} 21-\mathrm{N} 3-\mathrm{C} 13$ & $121.6(3)$ & $\mathrm{H} 26 \mathrm{~A}-\mathrm{C} 26-\mathrm{H} 26 \mathrm{~B}$ & 107.7 \\
\hline $\mathrm{C} 21-\mathrm{N} 3-\mathrm{C} 14$ & $119.9(3)$ & $\mathrm{C} 28-\mathrm{C} 27-\mathrm{C} 32$ & $118.6(5)$ \\
\hline $\mathrm{C} 13-\mathrm{N} 3-\mathrm{C} 14$ & $118.6(3)$ & $\mathrm{C} 28-\mathrm{C} 27-\mathrm{C} 26$ & $122.0(5)$ \\
\hline $\mathrm{C} 6-\mathrm{C} 1-\mathrm{C} 2$ & $117.7(4)$ & $\mathrm{C} 32-\mathrm{C} 27-\mathrm{C} 26$ & $119.4(5)$ \\
\hline $\mathrm{C} 6-\mathrm{C} 1-\mathrm{P} 1$ & $125.2(4)$ & $\mathrm{C} 27-\mathrm{C} 28-\mathrm{C} 29$ & $120.7(6)$ \\
\hline $\mathrm{C} 2-\mathrm{C} 1-\mathrm{P} 1$ & $117.1(4)$ & $\mathrm{C} 27-\mathrm{C} 28-\mathrm{H} 28$ & 119.7 \\
\hline $\mathrm{C} 3-\mathrm{C} 2-\mathrm{C} 1$ & $120.9(5)$ & $\mathrm{C} 29-\mathrm{C} 28-\mathrm{H} 28$ & 119.7 \\
\hline $\mathrm{C} 3-\mathrm{C} 2-\mathrm{H} 2$ & 119.5 & $\mathrm{C} 30-\mathrm{C} 29-\mathrm{C} 28$ & $121.3(7)$ \\
\hline $\mathrm{C} 1-\mathrm{C} 2-\mathrm{H} 2$ & 119.5 & $\mathrm{C} 30-\mathrm{C} 29-\mathrm{H} 29$ & 119.3 \\
\hline $\mathrm{C} 4-\mathrm{C} 3-\mathrm{C} 2$ & $120.3(5)$ & $\mathrm{C} 28-\mathrm{C} 29-\mathrm{H} 29$ & 119.3 \\
\hline
\end{tabular}




\begin{tabular}{|c|c|c|c|}
\hline $\mathrm{C} 4-\mathrm{C} 3-\mathrm{H} 3$ & 119.9 & $\mathrm{C} 29-\mathrm{C} 30-\mathrm{C} 31$ & $119.3(6)$ \\
\hline $\mathrm{C} 2-\mathrm{C} 3-\mathrm{H} 3$ & 119.9 & $\mathrm{C} 29-\mathrm{C} 30-\mathrm{H} 30$ & 120.4 \\
\hline $\mathrm{C} 3-\mathrm{C} 4-\mathrm{C} 5$ & $119.7(5)$ & $\mathrm{C} 31-\mathrm{C} 30-\mathrm{H} 30$ & 120.4 \\
\hline $\mathrm{C} 3-\mathrm{C} 4-\mathrm{H} 4$ & 120.2 & $\mathrm{C} 30-\mathrm{C} 31-\mathrm{C} 32$ & $120.2(6)$ \\
\hline $\mathrm{C} 5-\mathrm{C} 4-\mathrm{H} 4$ & 120.2 & $\mathrm{C} 30-\mathrm{C} 31-\mathrm{H} 31$ & 119.9 \\
\hline $\mathrm{C} 4-\mathrm{C} 5-\mathrm{C} 6$ & $121.3(5)$ & $\mathrm{C} 32-\mathrm{C} 31-\mathrm{H} 31$ & 119.9 \\
\hline $\mathrm{C} 4-\mathrm{C} 5-\mathrm{H} 5$ & 119.4 & $\mathrm{C} 27-\mathrm{C} 32-\mathrm{C} 31$ & $119.9(6)$ \\
\hline $\mathrm{C} 6-\mathrm{C} 5-\mathrm{H} 5$ & 119.4 & $\mathrm{C} 27-\mathrm{C} 32-\mathrm{H} 32$ & 120.0 \\
\hline $\mathrm{C} 5-\mathrm{C} 6-\mathrm{C} 1$ & $120.2(5)$ & $\mathrm{C} 31-\mathrm{C} 32-\mathrm{H} 32$ & 120.0 \\
\hline $\mathrm{C} 5-\mathrm{C} 6-\mathrm{H} 6$ & 119.9 & $\mathrm{~N} 1-\mathrm{C} 33-\mathrm{P} 2$ & $119.4(3)$ \\
\hline $\mathrm{C} 1-\mathrm{C} 6-\mathrm{H} 6$ & 119.9 & $\mathrm{~N} 1-\mathrm{C} 33-\mathrm{H} 33 \mathrm{~A}$ & 107.5 \\
\hline $\mathrm{C} 8-\mathrm{C} 7-\mathrm{C} 12$ & $119.2(4)$ & $\mathrm{P} 2-\mathrm{C} 33-\mathrm{H} 33 \mathrm{~A}$ & 107.5 \\
\hline $\mathrm{C} 8-\mathrm{C} 7-\mathrm{P} 1$ & $123.2(4)$ & $\mathrm{N} 1-\mathrm{C} 33-\mathrm{H} 33 \mathrm{~B}$ & 107.5 \\
\hline $\mathrm{C} 12-\mathrm{C} 7-\mathrm{P} 1$ & $117.1(3)$ & $\mathrm{P} 2-\mathrm{C} 33-\mathrm{H} 33 \mathrm{~B}$ & 107.5 \\
\hline $\mathrm{C} 7-\mathrm{C} 8-\mathrm{C} 9$ & $119.7(5)$ & $\mathrm{H} 33 \mathrm{~A}-\mathrm{C} 33-\mathrm{H} 33 \mathrm{~B}$ & 107.0 \\
\hline $\mathrm{C} 7-\mathrm{C} 8-\mathrm{H} 8$ & 120.1 & $\mathrm{C} 35-\mathrm{C} 34-\mathrm{C} 39$ & $117.6(4)$ \\
\hline $\mathrm{C} 9-\mathrm{C} 8-\mathrm{H} 8$ & 120.1 & $\mathrm{C} 35-\mathrm{C} 34-\mathrm{P} 2$ & $120.9(3)$ \\
\hline $\mathrm{C} 10-\mathrm{C} 9-\mathrm{C} 8$ & $120.0(6)$ & $\mathrm{C} 39-\mathrm{C} 34-\mathrm{P} 2$ & $121.4(3)$ \\
\hline $\mathrm{C} 10-\mathrm{C} 9-\mathrm{H} 9$ & 120.0 & $\mathrm{C} 34-\mathrm{C} 35-\mathrm{C} 36$ & $121.0(5)$ \\
\hline $\mathrm{C} 8-\mathrm{C} 9-\mathrm{H} 9$ & 120.0 & $\mathrm{C} 34-\mathrm{C} 35-\mathrm{H} 35$ & 119.5 \\
\hline $\mathrm{C} 11-\mathrm{C} 10-\mathrm{C} 9$ & $120.2(5)$ & $\mathrm{C} 36-\mathrm{C} 35-\mathrm{H} 35$ & 119.5 \\
\hline $\mathrm{C} 11-\mathrm{C} 10-\mathrm{H} 10$ & 119.9 & $\mathrm{C} 35-\mathrm{C} 36-\mathrm{C} 37$ & $119.4(5)$ \\
\hline $\mathrm{C} 9-\mathrm{C} 10-\mathrm{H} 10$ & 119.9 & $\mathrm{C} 35-\mathrm{C} 36-\mathrm{H} 36$ & 120.3 \\
\hline $\mathrm{C} 10-\mathrm{C} 11-\mathrm{C} 12$ & $120.9(6)$ & $\mathrm{C} 37-\mathrm{C} 36-\mathrm{H} 36$ & 120.3 \\
\hline $\mathrm{C} 10-\mathrm{C} 11-\mathrm{H} 11$ & 119.6 & $\mathrm{C} 38-\mathrm{C} 37-\mathrm{C} 36$ & $120.5(5)$ \\
\hline $\mathrm{C} 12-\mathrm{C} 11-\mathrm{H} 11$ & 119.6 & $\mathrm{C} 38-\mathrm{C} 37-\mathrm{H} 37$ & 119.8 \\
\hline $\mathrm{C} 11-\mathrm{C} 12-\mathrm{C} 7$ & $120.0(5)$ & $\mathrm{C} 36-\mathrm{C} 37-\mathrm{H} 37$ & 119.8 \\
\hline $\mathrm{C} 11-\mathrm{C} 12-\mathrm{H} 12$ & 120.0 & $\mathrm{C} 37-\mathrm{C} 38-\mathrm{C} 39$ & $119.8(5)$ \\
\hline $\mathrm{C} 7-\mathrm{C} 12-\mathrm{H} 12$ & 120.0 & $\mathrm{C} 37-\mathrm{C} 38-\mathrm{H} 38$ & 120.1 \\
\hline $\mathrm{N} 3-\mathrm{C} 13-\mathrm{P} 1$ & $115.9(3)$ & $\mathrm{C} 39-\mathrm{C} 38-\mathrm{H} 38$ & 120.1 \\
\hline N3-C13-H13A & 108.3 & $\mathrm{C} 38-\mathrm{C} 39-\mathrm{C} 34$ & $121.7(5)$ \\
\hline $\mathrm{P} 1-\mathrm{C} 13-\mathrm{H} 13 \mathrm{~A}$ & 108.3 & $\mathrm{C} 38-\mathrm{C} 39-\mathrm{H} 39$ & 119.1 \\
\hline $\mathrm{N} 3-\mathrm{C} 13-\mathrm{H} 13 \mathrm{~B}$ & 108.3 & $\mathrm{C} 34-\mathrm{C} 39-\mathrm{H} 39$ & 119.1 \\
\hline $\mathrm{P} 1-\mathrm{C} 13-\mathrm{H} 13 \mathrm{~B}$ & 108.3 & $\mathrm{C} 45-\mathrm{C} 40-\mathrm{C} 41$ & $118.3(4)$ \\
\hline $\mathrm{H} 13 \mathrm{~A}-\mathrm{C} 13-\mathrm{H} 13 \mathrm{~B}$ & 107.4 & $\mathrm{C} 45-\mathrm{C} 40-\mathrm{P} 2$ & $125.2(4)$ \\
\hline $\mathrm{N} 3-\mathrm{C} 14-\mathrm{C} 15$ & $113.8(3)$ & $\mathrm{C} 41-\mathrm{C} 40-\mathrm{P} 2$ & $116.5(3)$ \\
\hline $\mathrm{N} 3-\mathrm{C} 14-\mathrm{H} 14 \mathrm{~A}$ & 108.8 & $\mathrm{C} 40-\mathrm{C} 41-\mathrm{C} 42$ & $119.5(5)$ \\
\hline $\mathrm{C} 15-\mathrm{C} 14-\mathrm{H} 14 \mathrm{~A}$ & 108.8 & $\mathrm{C} 40-\mathrm{C} 41-\mathrm{H} 41$ & 120.3 \\
\hline N3- C14-H14B & 108.8 & $\mathrm{C} 42-\mathrm{C} 41-\mathrm{H} 41$ & 120.3 \\
\hline $\mathrm{C} 15-\mathrm{C} 14-\mathrm{H} 14 \mathrm{~B}$ & 108.8 & $\mathrm{C} 43-\mathrm{C} 42-\mathrm{C} 41$ & $121.1(6)$ \\
\hline $\mathrm{H} 14 \mathrm{~A}-\mathrm{C} 14-\mathrm{H} 14 \mathrm{~B}$ & 107.7 & $\mathrm{C} 43-\mathrm{C} 42-\mathrm{H} 42$ & 119.5 \\
\hline $\mathrm{C} 20-\mathrm{C} 15-\mathrm{C} 16$ & $117.7(4)$ & $\mathrm{C} 41-\mathrm{C} 42-\mathrm{H} 42$ & 119.5 \\
\hline $\mathrm{C} 20-\mathrm{C} 15-\mathrm{C} 14$ & $121.4(4)$ & $\mathrm{C} 42-\mathrm{C} 43-\mathrm{C} 44$ & $120.3(6)$ \\
\hline $\mathrm{C} 16-\mathrm{C} 15-\mathrm{C} 14$ & $120.7(5)$ & $\mathrm{C} 42-\mathrm{C} 43-\mathrm{H} 43$ & 119.8 \\
\hline $\mathrm{C} 17-\mathrm{C} 16-\mathrm{C} 15$ & $121.8(5)$ & $\mathrm{C} 44-\mathrm{C} 43-\mathrm{H} 43$ & 119.8 \\
\hline $\mathrm{C} 17-\mathrm{C} 16-\mathrm{H} 16$ & 119.1 & $\mathrm{C} 43-\mathrm{C} 44-\mathrm{C} 45$ & $120.3(6)$ \\
\hline $\mathrm{C} 15-\mathrm{C} 16-\mathrm{H} 16$ & 119.1 & $\mathrm{C} 43-\mathrm{C} 44-\mathrm{H} 44$ & 119.9 \\
\hline $\mathrm{C} 16-\mathrm{C} 17-\mathrm{C} 18$ & $119.0(6)$ & $\mathrm{C} 45-\mathrm{C} 44-\mathrm{H} 44$ & 119.9 \\
\hline
\end{tabular}




\begin{tabular}{|c|c|c|c|}
\hline $\mathrm{C} 16-\mathrm{C} 17-\mathrm{H} 17$ & 120.5 & $\mathrm{C} 40-\mathrm{C} 45-\mathrm{C} 44$ & $120.5(5)$ \\
\hline $\mathrm{C} 18-\mathrm{C} 17-\mathrm{H} 17$ & 120.5 & $\mathrm{C} 40-\mathrm{C} 45-\mathrm{H} 45$ & 119.7 \\
\hline $\mathrm{C} 19-\mathrm{C} 18-\mathrm{C} 17$ & $120.6(6)$ & $\mathrm{C} 44-\mathrm{C} 45-\mathrm{H} 45$ & 119.7 \\
\hline $\mathrm{C} 19-\mathrm{C} 18-\mathrm{H} 18$ & 119.7 & $\mathrm{Pt} 1-\mathrm{C} 46-\mathrm{H} 46 \mathrm{~A}$ & 109.5 \\
\hline $\mathrm{C} 17-\mathrm{C} 18-\mathrm{H} 18$ & 119.7 & $\mathrm{Pt} 1-\mathrm{C} 46-\mathrm{H} 46 \mathrm{~B}$ & 109.5 \\
\hline $\mathrm{C} 18-\mathrm{C} 19-\mathrm{C} 20$ & $120.5(6)$ & $\mathrm{H} 46 \mathrm{~A}-\mathrm{C} 46-\mathrm{H} 46 \mathrm{~B}$ & 109.5 \\
\hline $\mathrm{C} 18-\mathrm{C} 19-\mathrm{H} 19$ & 119.8 & $\mathrm{Pt} 1-\mathrm{C} 46-\mathrm{H} 46 \mathrm{C}$ & 109.5 \\
\hline $\mathrm{C} 20-\mathrm{C} 19-\mathrm{H} 19$ & 119.8 & $\mathrm{H} 46 \mathrm{~A}-\mathrm{C} 46-\mathrm{H} 46 \mathrm{C}$ & 109.5 \\
\hline $\mathrm{C} 15-\mathrm{C} 20-\mathrm{C} 19$ & $120.4(5)$ & $\mathrm{H} 46 \mathrm{~B}-\mathrm{C} 46-\mathrm{H} 46 \mathrm{C}$ & 109.5 \\
\hline $\mathrm{C} 46-\mathrm{Pt} 1-\mathrm{P} 1-\mathrm{C} 7$ & $34.8(2)$ & $\mathrm{C} 14-\mathrm{N} 3-\mathrm{C} 21-\mathrm{N} 2$ & $-174.1(3)$ \\
\hline $\mathrm{P} 2-\mathrm{Pt} 1-\mathrm{P} 1-\mathrm{C} 7$ & $-150.40(16)$ & $\mathrm{C} 13-\mathrm{N} 3-\mathrm{C} 21-\mathrm{C} 22$ & $-174.0(4)$ \\
\hline $\mathrm{C} 46-\mathrm{Pt} 1-\mathrm{P} 1-\mathrm{C} 1$ & $-89.30(18)$ & $\mathrm{C} 14-\mathrm{N} 3-\mathrm{C} 21-\mathrm{C} 22$ & $7.4(6)$ \\
\hline $\mathrm{P} 2-\mathrm{Pt} 1-\mathrm{P} 1-\mathrm{C} 1$ & $85.50(14)$ & $\mathrm{N} 2-\mathrm{C} 21-\mathrm{C} 22-\mathrm{C} 23$ & $-3.8(6)$ \\
\hline $\mathrm{C} 46-\mathrm{Pt} 1-\mathrm{P} 1-\mathrm{C} 13$ & $149.89(18)$ & $\mathrm{N} 3-\mathrm{C} 21-\mathrm{C} 22-\mathrm{C} 23$ & $174.7(4)$ \\
\hline $\mathrm{P} 2-\mathrm{Pt} 1-\mathrm{P} 1-\mathrm{C} 13$ & $-35.31(15)$ & $\mathrm{C} 21-\mathrm{C} 22-\mathrm{C} 23-\mathrm{C} 24$ & $-0.5(7)$ \\
\hline $\mathrm{P} 1-\mathrm{Pt} 1-\mathrm{P} 2-\mathrm{C} 40$ & $-8.50(19)$ & $\mathrm{C} 22-\mathrm{C} 23-\mathrm{C} 24-\mathrm{C} 25$ & $3.0(7)$ \\
\hline $\mathrm{C} 11-\mathrm{Pt} 1-\mathrm{P} 2-\mathrm{C} 40$ & $173.44(18)$ & $\mathrm{C} 21-\mathrm{N} 2-\mathrm{C} 25-\mathrm{N} 1$ & $177.0(3)$ \\
\hline $\mathrm{P} 1-\mathrm{Pt} 1-\mathrm{P} 2-\mathrm{C} 34$ & $-123.05(15)$ & $\mathrm{C} 21-\mathrm{N} 2-\mathrm{C} 25-\mathrm{C} 24$ & $-2.6(6)$ \\
\hline $\mathrm{C} 11-\mathrm{Pt} 1-\mathrm{P} 2-\mathrm{C} 34$ & $58.88(15)$ & $\mathrm{C} 33-\mathrm{N} 1-\mathrm{C} 25-\mathrm{N} 2$ & $19.7(5)$ \\
\hline $\mathrm{P} 1-\mathrm{Pt} 1-\mathrm{P} 2-\mathrm{C} 33$ & $123.31(17)$ & $\mathrm{C} 26-\mathrm{N} 1-\mathrm{C} 25-\mathrm{N} 2$ & $-158.0(4)$ \\
\hline $\mathrm{C} 11-\mathrm{Pt} 1-\mathrm{P} 2-\mathrm{C} 33$ & $-54.75(17)$ & $\mathrm{C} 33-\mathrm{N} 1-\mathrm{C} 25-\mathrm{C} 24$ & $-160.7(4)$ \\
\hline $\mathrm{C} 7-\mathrm{P} 1-\mathrm{C} 1-\mathrm{C} 6$ & $72.9(4)$ & $\mathrm{C} 26-\mathrm{N} 1-\mathrm{C} 25-\mathrm{C} 24$ & $21.6(6)$ \\
\hline $\mathrm{C} 13-\mathrm{P} 1-\mathrm{C} 1-\mathrm{C} 6$ & $-29.6(4)$ & $\mathrm{C} 23-\mathrm{C} 24-\mathrm{C} 25-\mathrm{N} 2$ & $-1.5(6)$ \\
\hline $\mathrm{Pt} 1-\mathrm{P} 1-\mathrm{C} 1-\mathrm{C} 6$ & $-157.6(4)$ & $\mathrm{C} 23-\mathrm{C} 24-\mathrm{C} 25-\mathrm{N} 1$ & $178.9(4)$ \\
\hline $\mathrm{C} 7-\mathrm{P} 1-\mathrm{C} 1-\mathrm{C} 2$ & $-108.8(4)$ & $\mathrm{C} 25-\mathrm{N} 1-\mathrm{C} 26-\mathrm{C} 27$ & $-118.3(5)$ \\
\hline $\mathrm{C} 13-\mathrm{P} 1-\mathrm{C} 1-\mathrm{C} 2$ & $148.7(3)$ & $\mathrm{C} 33-\mathrm{N} 1-\mathrm{C} 26-\mathrm{C} 27$ & $63.9(5)$ \\
\hline $\mathrm{Pt} 1-\mathrm{P} 1-\mathrm{C} 1-\mathrm{C} 2$ & $20.8(4)$ & $\mathrm{N} 1-\mathrm{C} 26-\mathrm{C} 27-\mathrm{C} 28$ & $56.3(6)$ \\
\hline $\mathrm{C} 6-\mathrm{C} 1-\mathrm{C} 2-\mathrm{C} 3$ & $0.4(7)$ & $\mathrm{N} 1-\mathrm{C} 26-\mathrm{C} 27-\mathrm{C} 32$ & $-125.6(5)$ \\
\hline $\mathrm{P} 1-\mathrm{C} 1-\mathrm{C} 2-\mathrm{C} 3$ & $-178.1(4)$ & $\mathrm{C} 32-\mathrm{C} 27-\mathrm{C} 28-\mathrm{C} 29$ & $0.6(8)$ \\
\hline $\mathrm{C} 1-\mathrm{C} 2-\mathrm{C} 3-\mathrm{C} 4$ & $0.1(8)$ & $\mathrm{C} 26-\mathrm{C} 27-\mathrm{C} 28-\mathrm{C} 29$ & $178.7(4)$ \\
\hline $\mathrm{C} 2-\mathrm{C} 3-\mathrm{C} 4-\mathrm{C} 5$ & $-0.5(9)$ & $\mathrm{C} 27-\mathrm{C} 28-\mathrm{C} 29-\mathrm{C} 30$ & $-1.0(8)$ \\
\hline $\mathrm{C} 3-\mathrm{C} 4-\mathrm{C} 5-\mathrm{C} 6$ & $0.5(10)$ & $\mathrm{C} 28-\mathrm{C} 29-\mathrm{C} 30-\mathrm{C} 31$ & $0.3(10)$ \\
\hline $\mathrm{C} 4-\mathrm{C} 5-\mathrm{C} 6-\mathrm{C} 1$ & $-0.1(9)$ & $\mathrm{C} 29-\mathrm{C} 30-\mathrm{C} 31-\mathrm{C} 32$ & $0.7(10)$ \\
\hline $\mathrm{C} 2-\mathrm{C} 1-\mathrm{C} 6-\mathrm{C} 5$ & $-0.4(7)$ & $\mathrm{C} 28-\mathrm{C} 27-\mathrm{C} 32-\mathrm{C} 31$ & $0.4(8)$ \\
\hline $\mathrm{P} 1-\mathrm{C} 1-\mathrm{C} 6-\mathrm{C} 5$ & $177.9(4)$ & $\mathrm{C} 26-\mathrm{C} 27-\mathrm{C} 32-\mathrm{C} 31$ & $-177.8(5)$ \\
\hline $\mathrm{C} 1-\mathrm{P} 1-\mathrm{C} 7-\mathrm{C} 8$ & $-21.8(4)$ & $\mathrm{C} 30-\mathrm{C} 31-\mathrm{C} 32-\mathrm{C} 27$ & $-1.0(9)$ \\
\hline $\mathrm{C} 13-\mathrm{P} 1-\mathrm{C} 7-\mathrm{C} 8$ & $86.5(4)$ & $\mathrm{C} 25-\mathrm{N} 1-\mathrm{C} 33-\mathrm{P} 2$ & $-51.3(5)$ \\
\hline $\mathrm{Pt} 1-\mathrm{P} 1-\mathrm{C} 7-\mathrm{C} 8$ & $-147.4(3)$ & $\mathrm{C} 26-\mathrm{N} 1-\mathrm{C} 33-\mathrm{P} 2$ & $126.5(4)$ \\
\hline $\mathrm{C} 1-\mathrm{P} 1-\mathrm{C} 7-\mathrm{C} 12$ & $166.9(3)$ & $\mathrm{C} 40-\mathrm{P} 2-\mathrm{C} 33-\mathrm{N} 1$ & $112.4(4)$ \\
\hline $\mathrm{C} 13-\mathrm{P} 1-\mathrm{C} 7-\mathrm{C} 12$ & $-84.7(4)$ & $\mathrm{C} 34-\mathrm{P} 2-\mathrm{C} 33-\mathrm{N} 1$ & $-148.1(4)$ \\
\hline $\mathrm{Pt} 1-\mathrm{P} 1-\mathrm{C} 7-\mathrm{C} 12$ & $41.4(4)$ & $\mathrm{Pt} 1-\mathrm{P} 2-\mathrm{C} 33-\mathrm{N} 1$ & $-27.9(4)$ \\
\hline $\mathrm{C} 12-\mathrm{C} 7-\mathrm{C} 8-\mathrm{C} 9$ & $1.5(7)$ & $\mathrm{C} 40-\mathrm{P} 2-\mathrm{C} 34-\mathrm{C} 35$ & $-114.5(4)$ \\
\hline $\mathrm{P} 1-\mathrm{C} 7-\mathrm{C} 8-\mathrm{C} 9$ & $-169.5(4)$ & $\mathrm{C} 33-\mathrm{P} 2-\mathrm{C} 34-\mathrm{C} 35$ & $138.4(4)$ \\
\hline $\mathrm{C} 7-\mathrm{C} 8-\mathrm{C} 9-\mathrm{C} 10$ & $-0.9(8)$ & $\mathrm{Pt} 1-\mathrm{P} 2-\mathrm{C} 34-\mathrm{C} 35$ & $16.6(4)$ \\
\hline $\mathrm{C} 8-\mathrm{C} 9-\mathrm{C} 10-\mathrm{C} 11$ & $0.4(9)$ & $\mathrm{C} 40-\mathrm{P} 2-\mathrm{C} 34-\mathrm{C} 39$ & $61.3(4)$ \\
\hline $\mathrm{C} 9-\mathrm{C} 10-\mathrm{C} 11-\mathrm{C} 12$ & $-0.7(9)$ & $\mathrm{C} 33-\mathrm{P} 2-\mathrm{C} 34-\mathrm{C} 39$ & $-45.8(4)$ \\
\hline $\mathrm{C} 10-\mathrm{C} 11-\mathrm{C} 12-\mathrm{C} 7$ & $1.4(8)$ & $\mathrm{Pt} 1-\mathrm{P} 2-\mathrm{C} 34-\mathrm{C} 39$ & $-167.7(3)$ \\
\hline
\end{tabular}




$\begin{array}{llll}\mathrm{C} 8-\mathrm{C} 7-\mathrm{C} 12-\mathrm{C} 11 & -1.8(7) & \mathrm{C} 39-\mathrm{C} 34-\mathrm{C} 35-\mathrm{C} 36 & -0.1(8) \\ \mathrm{P} 1-\mathrm{C} 7-\mathrm{C} 12-\mathrm{C} 11 & 169.8(4) & \mathrm{P} 2-\mathrm{C} 34-\mathrm{C} 35-\mathrm{C} 36 & 175.8(4) \\ \mathrm{C} 21-\mathrm{N} 3-\mathrm{C} 13-\mathrm{P} 1 & 73.8(5) & \mathrm{C} 34-\mathrm{C} 35-\mathrm{C} 36-\mathrm{C} 37 & 1.0(9) \\ \mathrm{C} 14-\mathrm{N} 3-\mathrm{C} 13-\mathrm{P} 1 & -107.6(4) & \mathrm{C} 35-\mathrm{C} 36-\mathrm{C} 37-\mathrm{C} 38 & -1.0(9) \\ \mathrm{C} 7-\mathrm{P} 1-\mathrm{C} 13-\mathrm{N} 3 & 47.1(3) & \mathrm{C} 36-\mathrm{C} 37-\mathrm{C} 38-\mathrm{C} 39 & 0.0(9) \\ \mathrm{C} 1-\mathrm{P} 1-\mathrm{C} 13-\mathrm{N} 3 & 157.5(3) & \mathrm{C} 37-\mathrm{C} 38-\mathrm{C} 39-\mathrm{C} 34 & 0.9(8) \\ \mathrm{P} 1-\mathrm{P} 1-\mathrm{C} 13-\mathrm{N} 3 & -78.9(3) & \mathrm{C} 35-\mathrm{C} 34-\mathrm{C} 39-\mathrm{C} 38 & -0.8(7) \\ \mathrm{C} 21-\mathrm{N} 3-\mathrm{C} 14-\mathrm{C} 15 & 74.5(5) & \mathrm{P} 2-\mathrm{C} 34-\mathrm{C} 39-\mathrm{C} 38 & -176.8(4) \\ \mathrm{C} 13-\mathrm{N} 3-\mathrm{C} 14-\mathrm{C} 15 & -104.2(4) & \mathrm{C} 34-\mathrm{P} 2-\mathrm{C} 40-\mathrm{C} 45 & -121.8(4) \\ \mathrm{N} 3-\mathrm{C} 14-\mathrm{C} 15-\mathrm{C} 20 & 32.0(6) & \mathrm{C} 33-\mathrm{P} 2-\mathrm{C} 40-\mathrm{C} 45 & -18.9(4) \\ \mathrm{N} 3-\mathrm{C} 14-\mathrm{C} 15-\mathrm{C} 16 & -151.7(4) & \mathrm{P} 1-\mathrm{P} 2-\mathrm{C} 40-\mathrm{C} 45 & 116.2(4) \\ \mathrm{C} 20-\mathrm{C} 15-\mathrm{C} 16-\mathrm{C} 17 & 0.6(6) & \mathrm{C} 34-\mathrm{P} 2-\mathrm{C} 40-\mathrm{C} 41 & 60.7(4) \\ \mathrm{C} 14-\mathrm{C} 15-\mathrm{C} 16-\mathrm{C} 17 & -175.9(5) & \mathrm{C} 33-\mathrm{P} 2-\mathrm{C} 40-\mathrm{C} 41 & 163.6(3) \\ \mathrm{C} 15-\mathrm{C} 16-\mathrm{C} 17-\mathrm{C} 18 & 0.0(8) & \mathrm{P} 1-\mathrm{P} 2-\mathrm{C} 40-\mathrm{C} 41 & -61.2(4) \\ \mathrm{C} 16-\mathrm{C} 17-\mathrm{C} 18-\mathrm{C} 19 & -1.0(9) & \mathrm{C} 45-\mathrm{C} 40-\mathrm{C} 41-\mathrm{C} 42 & -0.5(6) \\ \mathrm{C} 17-\mathrm{C} 18-\mathrm{C} 19-\mathrm{C} 20 & 1.3(9) & \mathrm{P} 2-\mathrm{C} 40-\mathrm{C} 41-\mathrm{C} 42 & 177.2(3) \\ \mathrm{C} 16-\mathrm{C} 15-\mathrm{C} 20-\mathrm{C} 19 & -0.2(6) & \mathrm{C} 40-\mathrm{C} 41-\mathrm{C} 42-\mathrm{C} 43 & 0.2(7) \\ \mathrm{C} 14-\mathrm{C} 15-\mathrm{C} 20-\mathrm{C} 19 & 176.2(4) & \mathrm{C} 41-\mathrm{C} 42-\mathrm{C} 43-\mathrm{C} 44 & 0.3(9) \\ \mathrm{C} 18-\mathrm{C} 19-\mathrm{C} 20-\mathrm{C} 15 & -0.7(8) & \mathrm{C} 42-\mathrm{C} 43-\mathrm{C} 44-\mathrm{C} 45 & -0.4(9) \\ \mathrm{C} 25-\mathrm{N} 2-\mathrm{C} 21-\mathrm{N} 3 & -173.3(4) & \mathrm{C} 41-\mathrm{C} 40-\mathrm{C} 45-\mathrm{C} 44 & 0.3(7) \\ \mathrm{C} 25-\mathrm{N} 2-\mathrm{C} 21-\mathrm{C} 22 & 5.2(5) & \mathrm{P} 2-\mathrm{C} 40-\mathrm{C} 45-\mathrm{C} 44 & -177.1(4) \\ \mathrm{C} 13-\mathrm{N} 3-\mathrm{C} 21-\mathrm{N} 2 & 4.5(6) & \mathrm{C} 43-\mathrm{C} 44-\mathrm{C} 45-\mathrm{C} 40 & 0.1(8)\end{array}$

Hydrogen-bond geometry $\left(A,{ }^{\circ}\right)$

$\mathrm{Cg} 1$ is the centroid of $\mathrm{C} 15-\mathrm{C} 20$ benzene ring.

\begin{tabular}{lllll}
\hline$D-\mathrm{H} \cdots A$ & $D-\mathrm{H}$ & $\mathrm{H} \cdots A$ & $D \cdots A$ & $D-\mathrm{H} \cdots A$ \\
\hline $\mathrm{C} 5-\mathrm{H} 5 \cdots C g 1^{\mathrm{i}}$ & 0.93 & 3.00 & $3.812(2)$ & 146 \\
$\mathrm{C} 26-\mathrm{H} 26 B \cdots C g 1^{\mathrm{ii}}$ & 0.97 & 2.96 & $3.807(2)$ & 147 \\
\hline
\end{tabular}

Symmetry codes: (i) $-x+3 / 2,-y+1, z+1 / 2$; (ii) $-x+3 / 2,-y+1, z-1 / 2$. 